future occupational health surveillance (OHS) and the role of OPs.

Methods First, the PubMed, EU-OSHA and International Labour Organisation databases were screened for literature from 2006 to August 31, 2016, concerning OHS, return-towork policies and risk management systems in Europe, Japan, Canada and other OECD countries. Second, a standardised mail survey was delivered to all members of the Belgian Professional Association of OPs.

Results

- The literature review showed that European directives legally inspired countries to adapt their legislations and develop strategic OHS plans. Nevertheless, the comparison of OHS in different countries shows a varied landscape.

- The response rate of the survey was $54 \%(n=262)$.

The results prompt a paradigm shift:

a. Within 5 years, OPs must focus more on return-to-work (96.3\%; 95\% CI: $93.2 \%$ to $98.1 \%$ ) and risk analyses $(84.9 \%$; 95\% CI: $79.9 \%$ to $88.8 \%$ ) while training occupational nurses (ONs) to execute routine occupational health examinations (46\%; 95\% CI: $39.8 \%$ to $52.4 \%$ ). Similarly, a more standardised and multidisciplinary approach to risk assessment should be developed (74.7\%; 95\% CI: $68.8 \%$ to $79.8 \%)$.

b. By 2030, OPs would be more specialised in toxicology, disability management, ionising radiation, etc. (42.2\%; 95\% CI: $36.2 \%$ to $48.5 \%$ ), and ONs would be trained on risk assessments (74.8\%; 95\% CI: $68.9 \%$ to $79.9 \%)$ and health promotion (98\%; 95\% CI: $95.3 \%$ to $99.1 \%)$. OPs disagree that general practitioners (GPs) could solely execute occupational health examinations $(81.7 \%$; $95 \% \mathrm{CI}$ : $76.4 \%$ to $86.1 \%)$.

Discussion The role of OPs must be part of a trend toward evidence-based OHS (i.e., studying the effectiveness and efficiency of 'fitness for the job assessments'), disability management and engagement with a wide network of stakeholders (such as ONs, GPs and health insurance organisations).

\section{RESPONSES FROM 'HIGH-STRESS' WORKERS OF THE STRESS CHECK PROGRAM IN JAPAN - A CASE STUDY}

H Tahara* , H Hiro. University of Occupational and Environmental Health, Japan, Kitakyushu, Japan

\subsection{6/oemed-2018-ICOHabstracts.488}

Introduction The Stress Check Program in Japan (SC), based on the Industrial Safety and Health Law, began on Dec 1, 2015. It requires an employer to

1. provide a survey of psychosocial stress for workers,

2. report to each individual worker his/her results,

3. arrange for an interview by a physician when requested to do so by a worker with high stress,

4. consider the opinions of the physician and improve working conditions for the worker, and

5. not take any action against the worker because he/she needs changes of his/her working conditions (Kawakami and Tsutsumi, 2016).

Ministry of Health, Labour and Welfare, Japan (MHLW) recommends an employer to assign an occupational health physician (OHP) to both the designated staff of the survey (DSS) and the interviewer (IVW).

Methods In June 2017, the Brief Job Stress Questionnaire (Shimomitsu, et al., 2000) was used as the survey at several offices of a company in Japan, where one of the presenters is assigned to OHP, DSS, and IVW. The criteria for 'high-stress' was based on an example appeared in the manual by MHLW. A presenter (as DSS) sent e-mail to fifty 'high-stress' workers, offering the interview by him (as IVW) and promoting permanent health consultation services by him (as OHP) or by outsourcing psychologists. Then he described the responses from the workers for a month.

Results Three workers requested the interview. Other ten workers reported his/her state of health by e-mail, phone or permanent health consultation. Another worker was accidentally arranged health consultation by the boss, not reported the result of the survey, due to sickness absence.

Conclusion Though SC as single program has weak scientific evidence for reducing workers' mental health problems; it may become more effective as a part of continuous occupational health promotion.

\section{PROCESS OF ACCREDITATION OF HEALTH SERVICE IN AUTOMOTIVE COMPANY OF BRAZIL}

${ }^{1}$ Paulo Zetola, ${ }^{2}$ Ana Claudia Soares, ${ }^{2}$ Livia Schwab, ${ }^{2}$ Jose Ari Bastos, ${ }^{2}$ Wilson Demarchi. ${ }^{1}$ Universidade Federal Do Paraná, Curitiba, Brazil; ${ }^{2}$ Medical Service Renault Automotive, Curitiba, Brazil

\subsection{6/oemed-2018-ICOHabstracts.489}

Introduction Private and public companies that have employees governed by the Consolidation of Labour Laws must have Specialised Services in Safety and Occupational Medicine, with the purpose of promoting health and protecting the integrity of the worker. These health professionals are responsible for urgent and emergency care in the work environment where we know the need for technical training of the professionals. The problem is that many find themselves without adequate updating in scientific training, updating in their internal processes, risk management that guarantees a safe assistance to the worker. These are the challenges of this research, identifying which are the main difficulties encountered by health managers of the company to implement an accreditation process in their medical services.

Method Cross-organisational study developed through the data and indicators obtained with the implementation of an Accreditation and Certification process of the National Accreditation Organisation (ONA) in Brazil, from 2014 to 2017.

Results The implementation of the Accreditation process in the company's Medical Service allowed us to identify many process failures in the service routines. Pharmacy, Ambulatory Infection, and Medical Records Committees were establish based on the requirements of the Accreditation and Certification Standards. All employees attended received an adhesive label with his name to be stitch to the clothing. Urgent care protocols were develop, as well as the Manchester Protocol for the prioritisation of care. A worksheet was create to identify all the Adverse Events found and a follow-up list of the pending action plans.

Discussion The Certification process is a significant differential for companies that are ready to implement it as it assures the patient's life and reduces the chances of medical failures or processes that generate damages to the patient. This company 